\title{
A Novel Coronavirus 2019 Linked with Pneumonia in China: Current Status and Future Prospects
}

\author{
Temoor Ahmed ${ }^{1}$, Muhammad Noman' ${ }^{1}$, Ahmad Almatroudi' ${ }^{2}$, Muhammad Shahid ${ }^{3}$, Mohsin \\ Khurshid ${ }^{4}$, Farheen Tariq ${ }^{5}$, Muhammad Tahir ul Qamar ${ }^{6}$, Rongrong $\mathbf{Y u}^{7}$, and Bin Li ${ }^{*}$ \\ 1 Institute of Biotechnology, Zhejiang University, 310058, Hangzhou, P.R. China; \\ temoorahmed248@gmail.com (T.A.); nomansiddique834@gmail.com (M.N.); libin0571@zju.edu.cn (B.L.) \\ 2 Department of Medical Laboratories, College of Applied Medical Sciences, Qassim University, Qassim \\ 51431, Saudi Arabia; aamtrody@qu.edu.sa (A.A.) \\ 3 Department of Bioinformatics and Biotechnology, Government College University, Faisalabad 38000, \\ Pakistan; mshahid@gcuf.edu.pk (M.S) \\ 4 Department of Microbiology, Government College University, Faisalabad 38000, Pakistan \\ mohsinkhurshid@gcuf.edu.pk (M.K) \\ 5 The Second Affiliated Hospital of Xi'an Jiaotong University, Xi'an, 710004 Shaanxi, P.R. China; \\ tariq.farheen@hotmail.com (F.T.) \\ 6 College of Life Science and Technology, Guangxi University, Nanning-530004, Guangxi Province, P.R. \\ China; m.tahirulqamar@hotmail.com (M.T) \\ 7 School of Education Science and Technology, Zhejiang University of Technology, Hangzhou 310032, P.R. \\ China; rrongyu@126.com (R.U) \\ Temoor Ahmed and Muhammad Noman contributed equally to this review article. \\ * Correspondence: mshahid@gcuf.edu.pk (M.S.); libin0571@zju.edu.cn (B.L.); \\ Tel: +92-300-7919822 (M.S.); +86-13515711458 (B.L.)
}

\begin{abstract}
The emergence of novel coronavirus strain 2019 (COVID-19) linked with pneumonia poses a serious threat to public health worldwide. Firstly, the COVID-19 was reported in Wuhan, Hubei Province, China in December 2019. Initially, the major proportion of virus-infected cases (i.e. about 99\%) was reported in China and now it is being reported in other counties as well. Humans begin to be infected within their communities and transmittance of the viral epidemic increased rapidly due to lack of understanding of its transmission routes and precautionary measures. The existence of COVID-19 in China threatened the population greatly due to the high incidence of fatal respiratory infections. Current investigations speculated that this virus transferred into a human from viral-infected bats. However, the process of interspecies viral transmission is an important scientific question to be addressed. Due to the continuous increase in the patients infected with COVID-19, the World Health Organization (WHO) has included this viral epidemic to the priority list of diseases. Therefore, accelerated research developments are required to control the spread of this outbreak, as it is declared as a public health emergency by WHO especially in the absence of efficacious drugs and vaccines. Our review encompasses the recent status of disease severity in China, a particular replication mechanism of COVID-19 and potential risks and precautionary measures required to avoid contact with this fatal viral infection.
\end{abstract}

Keywords: Coronavirus; China; Human; infectious diseases; Pneumonia

\section{Introduction}

Pneumonia linked with the novel strain of coronavirus 2019 (COVID-19) is the most incessant infectious disease in humans, particularly in China and is the leading cause of disturbing normal global public health [1]. Coronaviruses (CoVs) are a large family of viruses enclosed with singlestranded positive-sense RNA (ssRNA), known since the mid-1960s. These viruses are capable of 
infecting a large number of hosts and causing different respiratory, enteric, liver and systemic diseases [2]. Due to their unique replication mechanism, CoVs have a tendency to undergo recombination and mutation, which constantly enable them to acclimatize to new hosts and ecological niches [3,4]. The World Health Organization (WHO) estimates that virus-induced fatal respiratory infections are the main reason for death worldwide. Until 2003, little research data was available about these deadly viruses and only $10 \mathrm{CoVs}$ were known. However, another virus-induced respiratory infection called Severe Acute Respiratory Syndrome (SARS) was emerged in 2003, due to which 800 people of more than twelve states of Asia, Europe and America were died [5]. After the emergence of this new viral infection, several new CoVs were discovered [6]. According to the International Committee on Taxonomy of Viruses (ICTV), CoVs groups have been assigned to different genera namely Alpha-, Beta-, Gamma- and Delta-CoVs $[7,8]$. The birds and animals served as the reservoir for the emergence of most of the novel viral strains due to the habit of walking in flocks and their ability to fly long distances. Birds have the potential to transmit emerging viruses among themselves and to humans. The diversity of migratory routes and bird species in China can bring several pathogens to the country, including CoVs $[9,10]$. A role of public health should also be considered as it can contribute to studies on the origin of these viruses, such as the agents that cause respiratory failure linked with COVID-19, severe acute respiratory syndrome coronavirus (SARS$\mathrm{CoV}$ ) and Middle East respiratory syndrome coronavirus (MERS-CoV). In this study, we compile the current insights of COVID-19 study with special emphasis to enhanced surveillance and control the deadly infections in different regions of China.

\section{The occurrence of COVID-19}

The presence of COVID-19 in China was first reported on 29 December 2019 when numerous health service units registered clusters of patients with pneumonia of unknown reason that was epidemiologically associated with seafood and other aquatic animals sold in a local market in Wuhan, Hubei Province, China [11,12]. However, the primary source of this novel COVID-19 is not confirmed yet. In China, studies were carried out in Wuhan and other regions of the country to identify the agents responsible for respiratory failure and COVID-19 was detected in patients linked with pneumonia [13]. This virus was detected in the bronchoalveolar lavage fluid of three victims and confirmed by using whole-genome sequencing and direct PCR. The respiratory tract infection that has been caused by this virus was termed as novel coronavirus-infected pneumonia (NCIP). Phylogenetic investigation showed that COVID-19 falls into the genus Betacoronavirus, which consists of numerous coronaviruses ( including SARS-CoV, SARS-like $\mathrm{CoV}$, and others) diagnosed in humans, bats, and other wild animals [1]. Reports indicating infections in family members as well as in healthcare workers confirmed the possibility of human-to-human transmission, though the exact mode and extent of transmission are still unclear. On 21 January 2020, the WHO suggested that there was possibly a sustained human-to-human transmission of this virus [14]. As per report of 21 February 2020, the number of reported confirmed cases of COVID-19 have been reached up to 75569 in china, and in other regions 1200 confirmed cases have been reported e.g. 1 in Egypt, 1 in Nepal, 1 in Sri Lanka, 1 in Cambodia, 3 in India, 3 in Philippines, 5 in Iran, 8 in Taiwan, 8 in Canada, 9 in United Arab Emirates, 15 in United States of America, 16 in Vietnam, 17 in Australia, 22 in Malaysia, 35 in Thailand, 47 in Europe, 85 in Singapore, 93 in Japan, 204 in Republic of Korea, and 634 in International conveyance (Diamond Princess) [15]. The 25\% of the total confirmed cases reported in 
China have been classified as serious illness cases by Chinese health authorities (from Hubei Province: $16 \%$ severely ill, $5 \%$ critically ill, and $4 \%$ have died) (Fig. 1). This alarming situation gives rise to the threat that this virus may spread to the other parts of the world. It is predicted that further increase in the human cases suspected to be infected with COVID-19 can convert this epidemic situation to pandemic by transferring this virus to other nations.

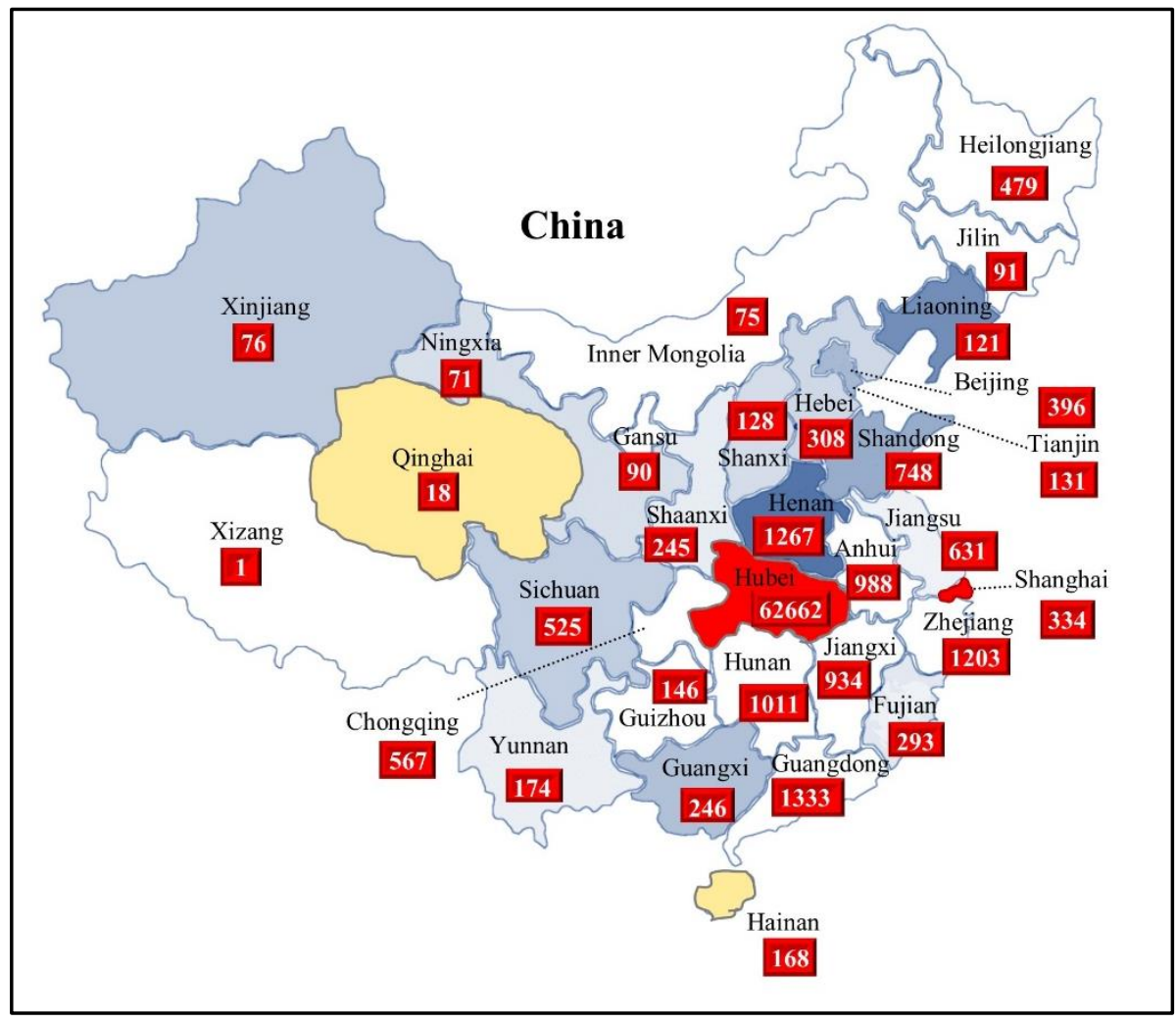

Fig. 1 Geographical distribution of confirmed cases of COVID-19 infection. As of 21 February 2020 [15].

\section{Respiratory diseases associated with COVID-19 and their diagnosis}

The possible relationship between human coronaviruses and respiratory diseases was identified when these viruses were isolated from the samples of patients with severe respiratory tract infections and subsequently observed for their mode of action in causing respiratory tract failure when inoculated into the volunteers. Currently, 2239 people have been reported to be died with WHO and NCIP in China (where 2144 were from Hubei province, 19 from Henan, 12 from Heilongjiang, 6 from Anhui, 6 from Chongqing, 5 from Guangdong, 5 from Hebei, 4 from Beijing, 4 from Hainan, 4 from Hunan, 4 from Shandong, 3 from Sichuan, 3 from Tianjin, 2 from Gansu, 2 from Guangxi, 2 from Hong Kong, 2 from Huizhou, 2 from Shanghai, 2 from Yunnan, 1 from Fujian, 1 from Jiangxi, 1 from Jilin, 1 from Liaoning, 1 from Shaanxi, 1 from Taiwan, 1 from Xinjiang, 1 from Zhejiang) and internationally 2 from International conveyance (Diamond Princess), 2 from Iran, 1 from France, 1 from Japan, 1 from Philippines, 1 from Republic of Korea. The majority of them were elderly or had compromised immune system due to other health complications according to the [15]. Li Lanjuan, director of the State Key Laboratory for Diagnosis and Treatment of Infectious Diseases, suggested some preventive measures to control pneumonia caused by the COVID-19. She said that the COVID19 infection in human has been linked to symptoms of upper and lower respiratory tract infections, 
which are being related to different clinical conditions such as fever, rhinorrhea, cough, and bronchiolitis. Therefore, the most important thing for us to identify is to take a throat swab and test for the virus. Now the technology is very advanced, as it can report whether you are infected with influenza A or B or the novel COVID-19. Currently, all hospitals of China are able to test new coronavirus and reagents are available in designated hospitals as previously described by [16].

\section{Classification of CoVs}

CoVs are classified in the order Nidovirales and family Coronaviridae, which includes the genera Coronavirus and Torovirus. Despite considerable differences in genetic complexity and viral architecture, viruses of the Coronaviridae, Arteviridae and Roniviridae family are characterized by a viral replication process that involves the production of multiple nested sub-genomic messenger RNAs (mRNAs) with 3'-like termination [17]. The organization chart of the taxonomy of the order Nidovirales is shown in Figure. 2. The Coronavirinae subfamily differs from the Torovirinae in viral morphology and genome size. The CoVs are capable of infecting humans and a wide variety of hosts, including other mammals (e.g. pigs, rats, dogs, horses, cattle, cetaceans, and bats) and birds (eg chickens, turkeys and pheasants), causing a variety of respiratory, enteric, liver and central nervous system dysfunction [18,19]. Recently, the CoV Study Group of the International Committee for Viral Taxonomy (ICTV) proposed replacing the traditional groups with the genera Alpha-, Beta-, Gammaand Delta-CoVs. Historically, the Alpha- and Beta-CoV groups were found in mammals, while GammaCoVs were found only in birds. However, some reports also suggest the presence of Gamma-CoVs in mammals $[8,20]$ and the presence of a fourth group called Delta-CoV was identified in birds and pigs [3].

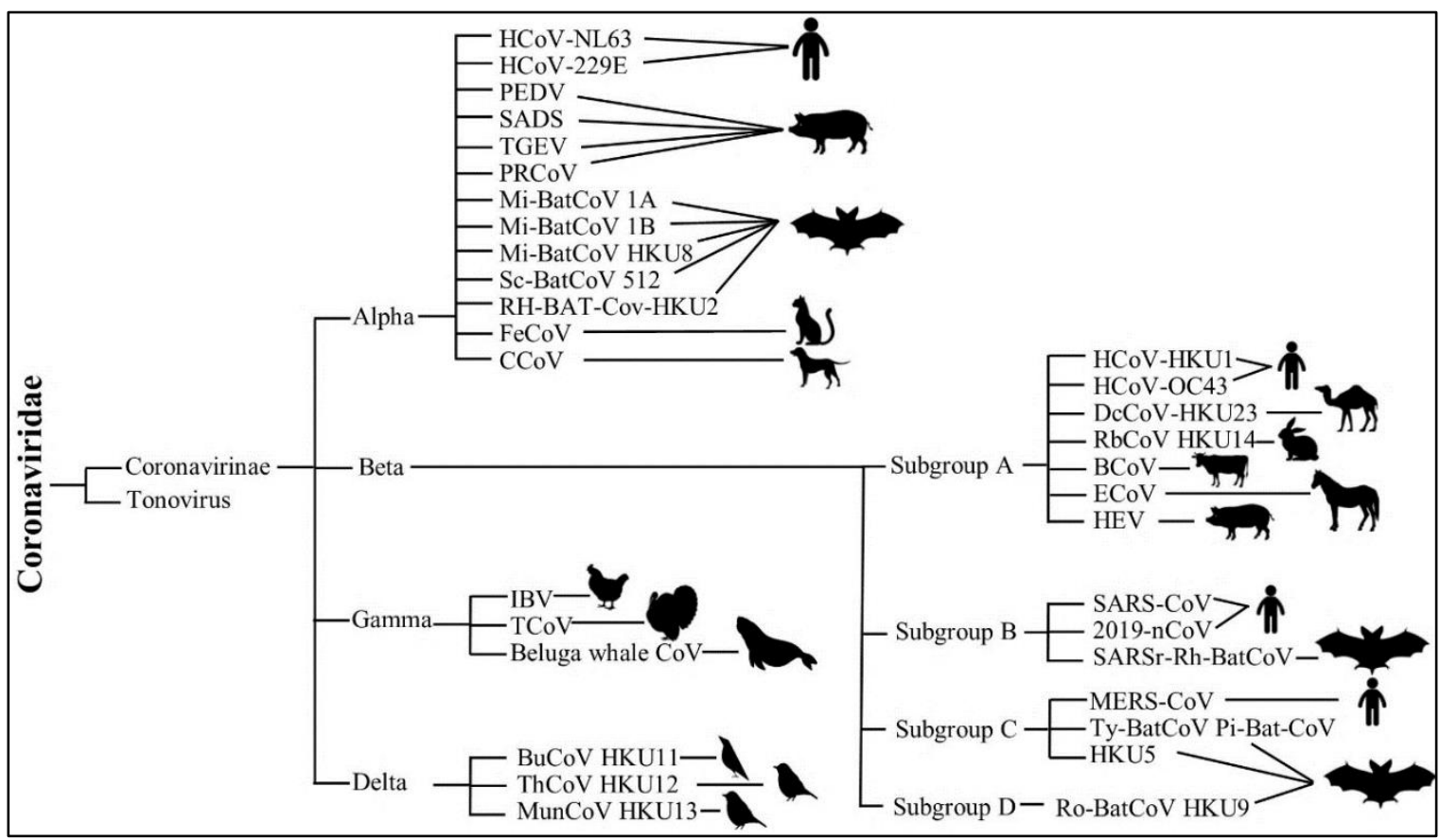

Figure. 2 Taxonomy of the Coronaviridae family.

\section{Morphology and genomic organization}

The CoV morphology is predominantly spherical, with a size of approximately 100 to $160 \mathrm{~nm}$ in diameter, however, they are quite pleomorphic due to the presence of an envelope which consists of 
a double layer of lipids and structural proteins [21,22]. The CoVs are single-stranded, non-segmented, polycistronic, positive polarity RNA (ssRNA) viruses presenting the major viral RNA genomes known to date $\left(27 \mathrm{to} 31 \mathrm{~kb}\right.$ ). In the genomic organization, 2/3 of the $5^{\prime}$ position of the genome contains 2 large ORFs ("open reading frames") called ORF1a and ORF1b, encoding non-structural proteins essential for RNA replication containing, for example, the RNA dependent RNA polymerase enzyme (RpRd) (Figure 3). However, the remaining 1/3 of the 3' proportion of the genome contains the genes encoding 4 proteins are originate: "Spike" (S), envelope (E), membrane (M) and nucleocapsid (N) [23]. The hemagglutinin esterase (HE) gene, characteristic of some Beta-CoVs, is located immediately after the S gene and is not present in Alpha- and Gamma-CoVs $[23,24]$. Before each ORF of the genome, there are intergenic spaces containing regulatory sequences called regulatory transcription sequences (TRS). The TRS is present in the 3' portion of the "leader" sequence and in the 5' portion of each of the "ORFs". These regulatory sequences are composed of a specific sequence of 6 to 10 nucleotides which are conserved among $\mathrm{CoVs}$ of the same group and participate in the transcription control of each viral gene [25]. The main properties linked to infectivity, virulence, and variability are associated with envelope proteins [26]. The spikes of the infectious coronavirus particle are oligomers of the $S$ glycoprotein, which bind the viral particle to the host cell receptor and can induce the fusion of the viral envelope with the cell membrane, being important for viral penetration into the host cell.

This protein can be divided into 3 structural domains between the $\mathrm{N}$-terminal and C-terminal regions. The external domain is subdivided into two subdomains (viz. S1 and S2), followed by a transmembrane domain and a small C-terminal cytoplasmic fragment. The S1 subdomain includes half of the N-terminal region and forms a globular portion of the spike that contains the sequences liable for binding to specific permissive cell receptors [27]. The M glycoprotein presents only a small fragment of the N-terminal domain exposed on the outside of the virions while being present mainly inside the viral particle. This protein participates in the arrangement of the viral particle, interacts with the $S$ and $N$ proteins, and may be associated with the determination of the intracellular binding site of the virus to the Golgi complex [28,29]. The E protein is the smallest protein in the viral envelope and has a function related to the beginning of the formation of the viral particle. Structural protein $\mathrm{N}$ is a 50-60 $\mathrm{kDa}$ phosphoprotein and has a strong connection with viral genomic RNA, composing the nucleocapsid through the involvement of the simple viral RNA strand. Protein N also interacts with protein $\mathrm{M}$, leading to the incorporation of the nucleocapsid in the viral particles, and can facilitate the formation of replicative RNA complexes, since its inhibition by antibodies can negatively influence the in vitro reaction of RNA polymerase [30]. 


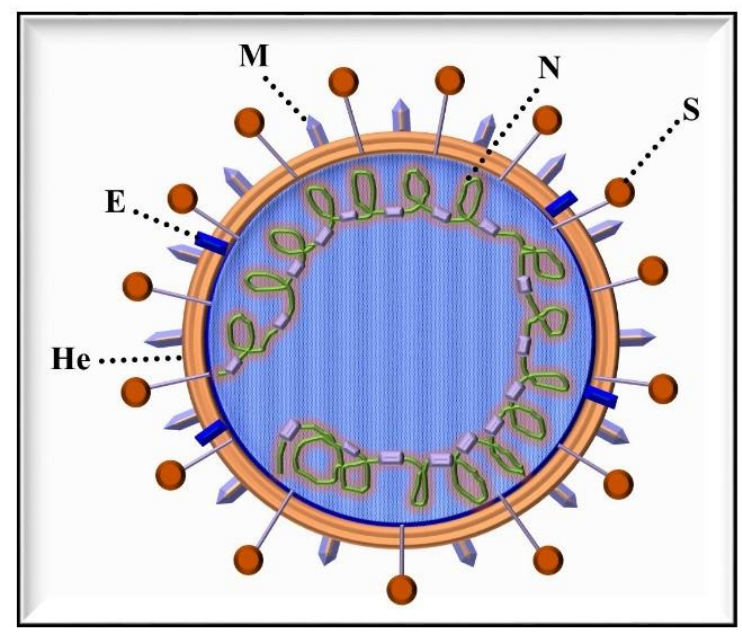

Figure. 3 Representation of the structural proteins that make up the coronavirus viral particle. In the nucleus, the protein N (nucleocapsid) is observed united to the simple RNA strand (complex RNAnucleocapsid protein). S-glycoproteins (Spike) are represented, HE (hemagglutinin-esterase), M (membrane glycoprotein) and protein E (envelope).

\section{CoV infectious cycle}

In spite of a variety of target tissues and host variety, CoV tropism is generally specific for epithelial cells in the respiratory or gastrointestinal tract. Viral replication occurs in the cytoplasm of the host cell [31]. Initially, the viral particle glycoprotein $S$ interacts with cellular receptors depending on the coronavirus strain. The spike protein can mediate the direct fusion of the virus with the cell membrane or the virus can penetrate the cell via receptor-mediated endocytosis and the $S$ protein induces the fusion of the viral envelope with the endosomal membrane, releasing the viral genomic RNA into the cytoplasm [32,33] (Figure. 4). The viral genome initially acts as an mRNA and the 5' region of the genome, composed of ORFs $1 \mathrm{a}$ and $1 \mathrm{~b}$ of the polymerase is directly translated by ribosomes into the polyprotein replicase (pp1ab). The translation of ORF $1 \mathrm{~b}$ is dependent on a change in the ribosomal frame reading codon, allowing the translation of proteins found in a different reading frame as compared to ORF 1a. The pp1a and pp1ab polyproteins are then processed by viral proteases (papain-like and 3CL proteases) generating 16 non-structural proteins (called nsp1-nsp16) components of the replication complex [34].

The 16 non-structural proteins are arranged with membranes generating double-membrane vesicles, where genomic RNA synthesis and subgenomic mRNA transcription occur. These RNAs conduct all proteins located after ORF1b and each subgenomic RNA has the same initial sequence ("leader") in the 5' portion, which is composed of 65 to 100 nucleotides depending on the virus type [35]. The leader sequence of each mRNA is similar to the "leader" sequence present exclusively at the beginning of the genomic RNA. Sub-genomic mRNAs are polycistronic structures, containing one to all the following ORFs of the gene to be expressed (except for RNA of the N gene), but only the first ORF of the subgenomic RNA located after the "leader" sequence is translated [35,36]. Subgenomic RNAs are then translated to generate structural proteins (S, M, N, E, HE) and accessory proteins. The number and function of proteins vary between different types of coronaviruses. The replicated genomic RNA forms a complex with the $\mathrm{N}$ protein forming the helical structure nucleocapsid. Structural virus proteins are inserted into the Endoplasmic Reticulum (ER). The nucleocapsid is then inserted into the intermediate compartment between the Golgi complex and the ER where the 
formation of the virion occurs. The viral particles are then incorporated into vesicles prior to their transport and release from the cell by exocytosis [36].

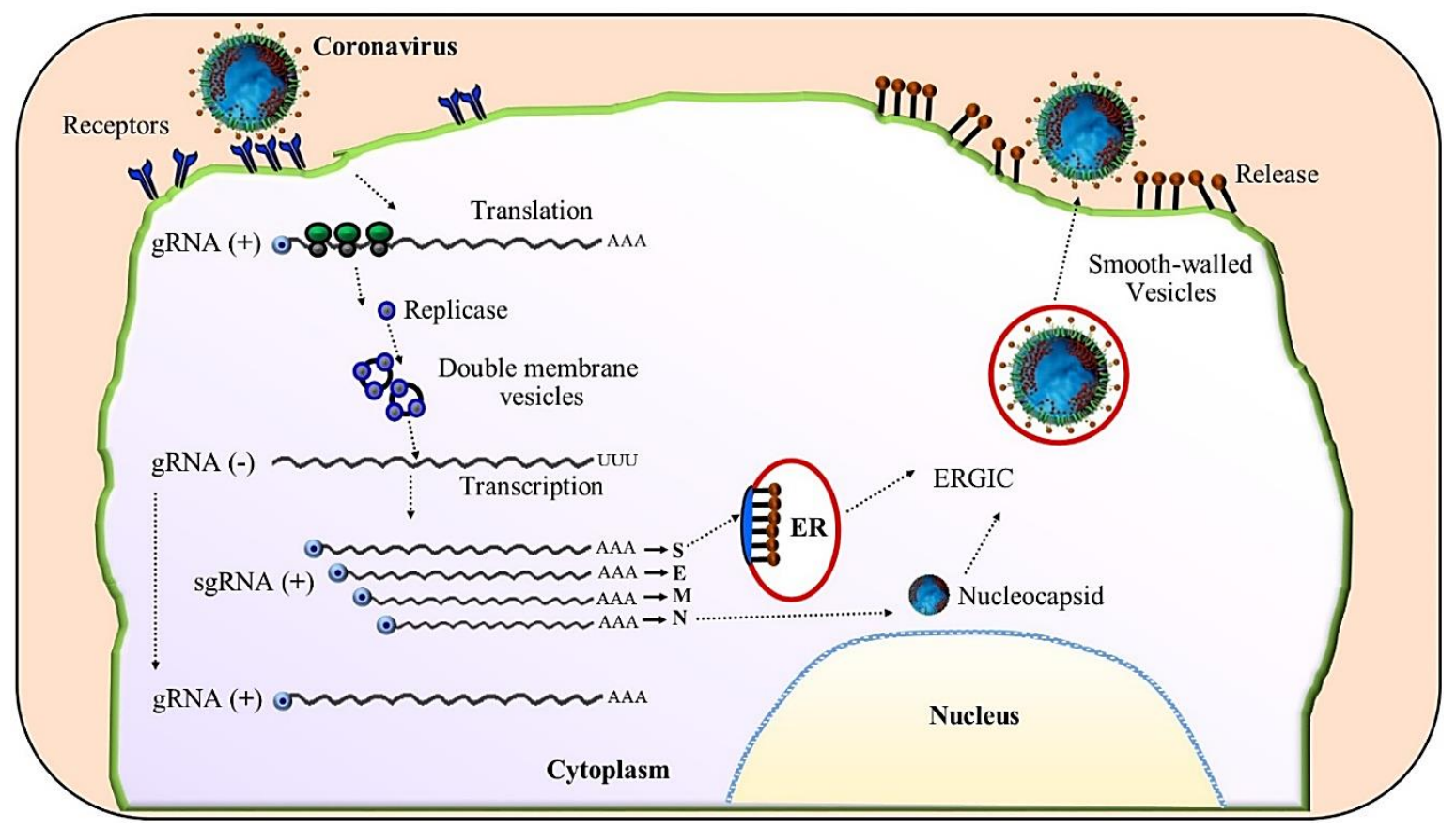

Figure. 4 Graphical representation of the $\mathrm{CoV}$ infectious cycle.

\section{Preventive measures}

Currently, there are no definite antiviral treatments for COVID-19 and the main treatment options available for COVID-19 are only supportive. Recombinant interferons (IFN) along with ribavirin have shown inadequate effects on CoV infection [37]. After the outbreak of SARS and MERS, plenty of anti-CoV agents have been developed to counter CoVs proteases, polymerases, MTases, and entry proteins, but so far, neither of them have been verified by clinical trials against COVID-19 $[38,39]$. Until now, the recommended primary treatment for CoVs is therapies with plasma and antibody gained from recovering patients. Several vaccination approaches for CoVs like inactivated viruses, live-attenuated viruses, viral vector-based vaccines, subunit vaccines, recombinant proteins, and DNA vaccines have been refined but still, these are only verified in animals [40,41]. Since there is neither practical remedy nor vaccines developed so far to counter COVID-19, the best approach against severe respiratory infection caused by novel $\mathrm{CoV}$ is to control the origin of infection, early diagnosis, and reporting, timely isolation of patients to avoid its spread and supportive treatments [42]. To discourage needless panic and fear among the public, epidemic data should be published on time and personal preventive measures like particular hand hygiene, avoiding crowds, wearing a surgical mask and proper ventilation should be prompted.

\section{Future prospects}

Due to the involvement of COVID-19, many facts regarding virulence, mode of transmission and the actual source of viral infection still need to be investigated. Up until now, the available data related to the viral outbreak is limited due to which there are many areas, which still required to be explored. For instance, the percent contribution of each route of transmission is not fully understood 
due to which the authorities face problems in controlling the infection spreading. However, the initial cases of COVID-19 were reported to have visited the Seafood Wholesale Market of Wuhan. Later, more COVID-19 cases emerged nearby the supermarkets of seafood located in the two districts of the city [43].

Furthermore, human-to-human transmission has been confirmed as family clusters within and outside China and health-care workers were found to be infected with COVID-19. However, with currently available data it is very difficult to explain/measure the extent of human-to-human transmission. According to a recent report, about 12,335 cases have been documented outside the Hubei province of China due to which it can be concluded that strong human-to-human viral transmission is occurring in Hubei and other provinces of China [44].

With the estimation of reproductive number (i.e. 1.4-2.5), the Chinese authorities suggested that the COVID-19 has higher transmissibility than MERS (reproductive number=0.7) but similar to SARS (reproductive number $=2-3$ ). The preliminary analysis further confirms the reproductive number of the virus estimated by Chinese authorities [45]. Further work has been done to measure the importation of risk to other cities of China and outside China [46]. The currently limited data is mainly focused on the mobility pattern with the least emphasis on the human-to-human viral transmission route [47].

The responsible Chinese authorities are performing their best to limit the transmission/spreading of the outbreak and implemented different disease preventive measures such as restrict people from New Lunar Year celebrations, prohibited public gathering in theatres and parks and also lock down public transport facilities particularly in Wuhan [44]. In addition, the authorities executed strict exit screening within and outside of Hubei province. However, research studies are required to understand the molecular basis of pathogenicity/virulence of COVID-19linked infection, which ultimately will help to identify the potential targets for antiviral medicines.

Author Contributions: TA, MN, MS, and BL made a significant contribution to conceived the study. All authors participated in the study design and drafted the manuscript.

Funding: This research work is financially supported by PhosAgro/UNESCO/IUPAC research grant \# 82. .

Acknowledgments: The authors would like to thank Dr. Mahmood Ahmad, Dr. Natasha Manzoor and Mr. Fahad Shahzad for English editing and data collection.

Conflicts of Interest: The authors declare no conflicts of interest.

\section{References}

1. Zhu, N.; Zhang, D.; Wang, W.; Li, X.; Yang, B.; Song, J.; Zhao, X.; Huang, B.; Shi, W.; Lu, R. A Novel Coronavirus from Patients with Pneumonia in China, 2019. New Engl. J. Med. 2020.

2. Lu, G.; Wang, Q.; Gao, G.F. Bat-to-human: spike features determining ‘host jump' of coronaviruses SARSCoV, MERS-CoV, and beyond. Trends Microbiol. 2015, 23, 468-478.

3. Woo, P.C.; Lau, S.K.; Lam, C.S.; Lau, C.C.; Tsang, A.K.; Lau, J.H.; Bai, R.; Teng, J.L.; Tsang, C.C.; Wang, M. Discovery of seven novel Mammalian and avian coronaviruses in the genus deltacoronavirus supports bat coronaviruses as the gene source of alphacoronavirus and betacoronavirus and avian coronaviruses as the gene source of gammacoronavirus and deltacoronavirus. J. Virol. 2012, 86, 3995-4008.

4. Wang, X.; Rong, W. Predicting acute respiratory infection in Chinese healthy individuals: A effective way of patient care. J. King Saud Univ. Sci 2020, 32, 1065-1068. 
5. WHO. Consensus document on the epidemiology of severe acute respiratory syndrome (SARS). https://apps.who.int/iris/handle/10665/70863 2003.

6. Woo, P.; Huang, Y.; Lau, S.; Yuen, K. Coronavirus genomics and bioinformatics analysis. Viruses. 2: 18041820. 2010.

7. van Regenmortel, M.H.; Fauquet, C.M.; Bishop, D.H.; Carstens, E.; Estes, M.; Lemon, S.; Maniloff, J.; Mayo, M.; McGeoch, D.; Pringle, C. Virus taxonomy: classification and nomenclature of viruses. Seventh report of the International Committee on Taxonomy of Viruses; Academic Press: 2000.

8. Adams, M.J.; Carstens, E. Ratification vote on taxonomic proposals to the International Committee on Taxonomy of Viruses (2012). Arch. Virol. 2012, 157, 1411-1422.

9. Wang, L.-F.; Walker, P.J.; Poon, L.L. Mass extinctions, biodiversity and mitochondrial function: are bats 'special'as reservoirs for emerging viruses? Curr. Opin. Virol. 2011, 1, 649-657.

10. Mackenzie, J.S.; Jeggo, M. Reservoirs and vectors of emerging viruses. Curr. Opin. Virol. 2013, 3, 170-179.

11. Read, J.M.; Bridgen, J.R.; Cummings, D.A.; Ho, A.; Jewell, C.P. Novel coronavirus 2019-nCoV: early estimation of epidemiological parameters and epidemic predictions. medRxiv. 2020.

12. MacIntyre, C.R. Wuhan novel coronavirus 2019nCoV. Global Biosecurity. 2020, 1.

13. Corman, V.M.; Landt, O.; Kaiser, M.; Molenkamp, R.; Meijer, A.; Chu, D.K.; Bleicker, T.; Brünink, S.; Schneider, J.; Schmidt, M.L. Detection of 2019 novel coronavirus (2019-nCoV) by real-time RT-PCR. Eurosurveillance 2020, 25, 2000045.

14. WHO. Twitter post. 21 Jan 2020. World Health Organization https://twitter.com/WHOWPRO/status/1219478547644813312 2020.

15. WHO 21 Feb 2020. W. https://www.who.int/docs/default-source/coronaviruse/situationreports/20200221-sitrep-32-covid-19.pdf?sfvrsn=4802d089_2020.

16. CGTN. Expert explains how to distinguish a common cold from novel pneumonia. https://news.cgtn.com/news/2020-01-26/Expert-explains-how-to-distinguish-a-common-cold-from-novelpneumonia-NzCxgxjLzi/index.html 2020.

17. Zhong, Y.; Tan, Y.W.; Liu, D.X. Recent progress in studies of arterivirus-and coronavirus-host interactions. Viruses. 2012, 4, 980-1010.

18. Gavier-Widén, D.; Meredith, A.; Duff, J.P. Infectious diseases of wild mammals and birds in Europe; John Wiley \& Sons: 2012.

19. Geldenhuys, M. Detection and characterization of coronaviruses from African bat species. University of Pretoria, 2013.

20. Woo, P.C.; Lau, S.K.; Yip, C.C.; Huang, Y.; Yuen, K.-Y. More and more coronaviruses: human coronavirus HKU1. Viruses 2009, 1, 57-71.

21. Fan, H.; Ooi, A.; Tan, Y.W.; Wang, S.; Fang, S.; Liu, D.X.; Lescar, J. The nucleocapsid protein of coronavirus infectious bronchitis virus: crystal structure of its N-terminal domain and multimerization properties. Structure 2005, 13, 1859-1868.

22. Hu, D.; Zhu, C.; Ai, L.; He, T.; Wang, Y.; Ye, F.; Yang, L.; Ding, C.; Zhu, X.; Lv, R. Genomic characterization and infectivity of a novel SARS-like coronavirus in Chinese bats. Emerg. Microbes. Infec. 2018, 7, 1-10.

23. Brian, D.; Baric, R. Coronavirus genome structure and replication. In Coronavirus replication and reverse genetics, Springer: 2005; pp. 1-30.

24. Van Der Hoek, L.; Pyrc, K.; Berkhout, B. Human coronavirus NL63, a new respiratory virus. FEMS Microbiol. Rev. 2006, 30, 760-773. 
25. Sawicki, S.G.; Sawicki, D.L.; Siddell, S.G. A contemporary view of coronavirus transcription. J. Virol. 2007, 81, 20-29.

26. Fan, H.; Ooi, A.; Tan, Y.W.; Wang, S.; Fang, S.; Liu, D.X.; Lescar, J. The nucleocapsid protein of coronavirus infectious bronchitis virus: crystal structure of its $\mathrm{N}$-terminal domain and multimerization properties. Structure. 2005, 13, 1859-1868.

27. Tripp, R.A.; Haynes, L.M.; Moore, D.; Anderson, B.; Tamin, A.; Harcourt, B.H.; Jones, L.P.; Yilla, M.; Babcock, G.J.; Greenough, T. Monoclonal antibodies to SARS-associated coronavirus (SARS-CoV): identification of neutralizing and antibodies reactive to S, N, M and E viral proteins. J. Virol. Methods. 2005, $128,21-28$.

28. Xue, B.; Blocquel, D.; Habchi, J.; Uversky, A.V.; Kurgan, L.; Uversky, V.N.; Longhi, S. Structural disorder in viral proteins. Chem. Rev. 2014, 114, 6880-6911.

29. Jayaram, H.; Fan, H.; Bowman, B.R.; Ooi, A.; Jayaram, J.; Collisson, E.W.; Lescar, J.; Prasad, B.V. X-ray structures of the $\mathrm{N}$-and C-terminal domains of a coronavirus nucleocapsid protein: implications for nucleocapsid formation. J. Virol. 2006, 80, 6612-6620.

30. Ababneh, M.M. Immune response to nucleocapsid protein of turkey coronavirus and its protective efficacy in turkeys. Purdue University, 2005.

31. Wevers, B.A.; van der Hoek, L. Recently discovered human coronaviruses. Clin. Lab. Med. 2009, 29, 715-724.

32. Marzi, A.; Gramberg, T.; Simmons, G.; Möller, P.; Rennekamp, A.J.; Krumbiegel, M.; Geier, M.; Eisemann, J.; Turza, N.; Saunier, B. DC-SIGN and DC-SIGNR interact with the glycoprotein of Marburg virus and the S protein of severe acute respiratory syndrome coronavirus. J. Virol. 2004, 78, 12090-12095.

33. Belouzard, S.; Millet, J.K.; Licitra, B.N.; Whittaker, G.R. Mechanisms of coronavirus cell entry mediated by the viral spike protein. Viruses. 2012, 4, 1011-1033.

34. van Boheemen, S.; de Graaf, M.; Lauber, C.; Bestebroer, T.M.; Raj, V.S.; Zaki, A.M.; Osterhaus, A.D.; Haagmans, B.L.; Gorbalenya, A.E.; Snijder, E.J. Genomic characterization of a newly discovered coronavirus associated with acute respiratory distress syndrome in humans. MBio 2012, 3, e00473-00412.

35. Imbert, I.; Snijder, E.J.; Dimitrova, M.; Guillemot, J.-C.; Lécine, P.; Canard, B. The SARS-Coronavirus PLnc domain of nsp3 as a replication/transcription scaffolding protein. Virus Res. 2008, 133, 136-148.

36. Baker, S.C. Coronaviruses: from common colds to severe acute respiratory syndrome. The Pediatric infectious disease journal 2004, 23, 1049-1050.

37. Cinatl, J.; Morgenstern, B.; Bauer, G.; Chandra, P.; Rabenau, H.; Doerr, H. Treatment of SARS with human interferons. The Lancet 2003, 362, 293-294.

38. Chan, J.F.; Chan, K.-H.; Kao, R.Y.; To, K.K.; Zheng, B.-J.; Li, C.P.; Li, P.T.; Dai, J.; Mok, F.K.; Chen, H. Broadspectrum antivirals for the emerging Middle East respiratory syndrome coronavirus. J. Infect. 2013, 67, 606616.

39. Cheng, K.-W.; Cheng, S.-C.; Chen, W.-Y.; Lin, M.-H.; Chuang, S.-J.; Cheng, I.-H.; Sun, C.-Y.; Chou, C.-Y. Thiopurine analogs and mycophenolic acid synergistically inhibit the papain-like protease of Middle East respiratory syndrome coronavirus. Antiviral Res. 2015, 115, 9-16.

40. Mair-Jenkins, J.; Saavedra-Campos, M.; Baillie, J.K.; Cleary, P.; Khaw, F.-M.; Lim, W.S.; Makki, S.; Rooney, K.D.; Group, C.P.S.; Nguyen-Van-Tam, J.S. The effectiveness of convalescent plasma and hyperimmune immunoglobulin for the treatment of severe acute respiratory infections of viral etiology: a systematic review and exploratory meta-analysis. J. Infect. Dis. 2015, 211, 80-90.

41. de Wit, E.; van Doremalen, N.; Falzarano, D.; Munster, V.J. SARS and MERS: recent insights into emerging coronaviruses. Nat. Rev. Microbiol. 2016, 14, 523. 
42. Chen, Y.; Liu, Q.; Guo, D. Coronaviruses: genome structure, replication, and pathogenesis. J. Med. Virol. 2020.

43. People.cn. \#New coronavirus pneumonia latency.

https://s.weibo.com/weibo?q=\%23\%E6\%96\%B0\%E5\%9E\%8B\%E5\%86\%A0\%E7\%8A\%B6\%E7\%97\%85\%E6\% AF\%92\%E8\%82\%BA\%E7\%82\%8E\%E6\%BD\%9C\%E4\%BC\%8F\%E6\%9C\%9F\%23Efrom=default 2020.

44. ECDC. Outbreak of acute respiratory syndrome associated with a novel coronavirus, China; First cases imported in the EU/EEA; second update 26 January https://www.ecdc.europa.eu/en/publicationsdata/risk-assessment-outbreak-acute-respiratory-syndrome-associated-novel-0 2020.

45. Read, J.M.; Bridgen, J.R.; Cummings, D.A.; Ho, A.; Jewell, C.P. Novel coronavirus 2019-nCoV: early estimation of epidemiological parameters and epidemic predictions. medRxiv 2020.

46. Imai N, C.A., Dorigatti I, Baguelin M, Donnelly CA, Riley S, et al. Transmissibility of 2019-nCoV 2020. https://www.imperial.ac.uk/mrc-global-infectious-disease-analysis/news--wuhan-coronavirus/. 2020.

47. Gralinski, L.E.; Menachery, V.D. Return of the Coronavirus: 2019-nCoV. Viruses. 2020, 12, 135. 